\title{
Current Status of Quark Gluon Plasma Signals
}

\author{
D. Zschiesche ${ }^{1}$, S. Bass ${ }^{2,3}$, M. Bleicher ${ }^{1}$, J. Brachmann ${ }^{1}$, L. Gerland ${ }^{1}$, \\ K. Paech ${ }^{1}$, S. Scherer ${ }^{1}$, S. Soff ${ }^{4}$, C. Spieles ${ }^{1}$, H. Weber ${ }^{1}$, H. Stöcker ${ }^{1}$, \\ W. Greiner ${ }^{1}$ \\ ${ }^{1}$ Institut für Theoretische Physik, J.W. Goethe Universität \\ 60054 Frankfurt a.M., Germany \\ 2 Department of Physics, Duke University \\ 27708-0305 Durham, NC, USA \\ ${ }^{3}$ RIKEN BNL Research Center, Brookhaven National Laboratory \\ Upton, NY 11973, USA \\ ${ }^{4}$ Physics Department, Brookhaven National Laboratory \\ Upton, NY 11973, USA
}

\begin{abstract}
Compelling evidence for the creation of a new form of matter has been claimed to be found in $\mathrm{Pb}+\mathrm{Pb}$ collisions at SPS. We discuss the uniqueness of often proposed experimental signatures for quark matter formation in relativistic heavy ion collisions. It is demonstrated that so far none of the proposed signals like $J / \psi$ meson production/suppression, strangeness enhancement, dileptons, and directed flow unambigiously show that a phase of deconfined matter has been formed in SPS Pb+Pb collisions. We emphasize the need for systematic future measurements to search for simultaneous irregularities in the excitation functions of several observables in order to come close to pinning the properties of hot, dense QCD matter from data.
\end{abstract}

\section{Introduction}

In the last few years researchers at Brookhaven and CERN have succeeded to measure a wide spectrum of observables with heavy ion beams, $A u+A u$ and $P b+P b$. While these programs continue to measure with greater precision the beam energy-, nuclear size-, and centrality dependence of those observables, it is important to recognize the major milestones passed thusfar in that work. The experiments have conclusively demonstrated the existence of strong nuclear $A$ dependence of, among others, $J / \psi$ and $\psi^{\prime}$ meson production and suppression, strangeness enhancement, hadronic resonance production, stopping and directed collective transverse and longitudinal flow of baryons and mesons - in and out of 
the impact plane, both at AGS and SPS energies -, and dilepton-enhancement below and above the $\rho$ meson mass. These observations support that a novel form of "resonance matter" at high energy- and baryon density has been created in nuclear collisions. The global multiplicity and transverse energy measurements prove that substantially more entropy is produced in $A+A$ collisions at the SPS than simple superposition of $A \times p p$ would imply. Multiple initial and final state interactions play a critical role in all observables. The high midrapidity baryon density (stopping) and the observed collective transverse and directed flow patterns constitute one of the strongest evidence for the existence of an extended pe$\operatorname{riod}(\Delta \tau \approx 10 \mathrm{fm} / \mathrm{c})$ of high pressure and strong final state interactions. The enhanced $\psi^{\prime}$ suppression in $S+U$ relative to $p+A$ also attests to this fact. The anomalous low mass dilepton enhancement shows that substantial in-medium modifications of multiple collision dynamics exist, probably related to in-medium collisional broadening of vector mesons. The non-saturation of the strangeness (and anti-strangeness) production shows that novel non-equilibrium production processes arise in these reactions. Finally, the centrality dependence of $J / \psi$ absorption in $P b+P b$ collisions presents further hints towards the nonequilibrium nature of such reactions. Is there evidence for the long sought-after quark-gluon plasma that thusfar has only existed as a binary array of predictions inside teraflop computers?

As we will discuss, it is too early to tell. Theoretically there are still too many "scenarios" and idealizations to provide a satisfactory answer. Recent results from microscopic transport models as well as macroscopic hydrodynamical calculations differ significantly from predictions of simple thermal models, e. g. in the flow pattern. Still, these nonequilibrium models provide reasonable predictions for the experimental data. We may therefore be forced to rethink our concept of what constitutes the deconfined phase in ultrarelativistic heavy-ion collisions. Most probably it is not a blob of thermalized quarks and gluons. Hence, a quark-gluon plasma can only be the source of differences to the predictions of these models for hadron ratios, the $J / \psi$ meson production, dilepton yields, or the excitation function of transverse flow. And there are experimental gaps such as the lack of intermediate mass $A \approx 100$ data and the limited number of beam energies studied thusfar, in particular between the AGS and SPS. Now the field is at the doorstep of the next milestone: $A+A$ at $\sqrt{s}=30-200 \mathrm{AGeV}$ which have started a few months ago.

\section{2. $\mathrm{J} / \psi$ production}

The QCD factorization theorem is used to evaluate the PQCD cross sections of heavy quarkonium interactions with ordinary hadrons. However, the charmonium states (here denoted $X$ ) are not sufficiently small to ignore nonperturbative QCD physics. Thus, we evaluate the nonperturbative QCD contribution to the cross sections of charmonium-nucleon interaction by using an interpolation between known cross sections [3]. The $J / \psi-N$ cross section evaluated in this paper is in reasonable agreement with SLAC data [

Indeed, the $A$-dependence of the $J / \psi$ production studied at SLAC at $E_{\text {inc }} \sim 20 \mathrm{GeV}$ exhibits a significant absorption effect [ [4] leading to $\sigma_{a b s}(J / \psi-N)=3.5 \pm 0.8 \mathrm{mb}$. It was demonstrated [ [5] that, in the kinematic region at SLAC, the color coherence effects 
are still small on the internucleon scale for the formation of $J / \psi$ 's. So, in contrast to the findings at higher energies, at intermediate energies this process measures the genuine $J / \psi-N$ interaction cross section at energies of $\sim 15-20 \mathrm{GeV}[$ [ $]$ ].

To evaluate the nonperturbative QCD contribution we use an interpolation formula [ 3] for the dependence of the cross section on the transverse size $b$ of a quark-gluon configuration Three reference points are used to fix our parametrization of the cross sections (cf. Tab. 11. The $X-N$ cross sections is calculated via: $\sigma=\int \sigma(b) \cdot|\Psi(x, y, z)|^{2} \mathrm{~d} x \mathrm{~d} y \mathrm{~d} z$, where $\Psi(x, y, z)$ is the charmonium wave function. In our calculations we use the wave functions from a non-relativistic charmonium model (see [ 6 ]).

Table 1. The total quarkonium-nucleon cross sections $\sigma$. For the $\chi$ two values arise, due to the spin dependent wave functions $(l m=10,11)$

\begin{tabular}{c|c|c|c|c|}
\hline$c \bar{m}=10,11$ & \\
$c \bar{c}$-state & $\mathrm{J} / \psi$ & $\psi^{\prime}$ & $\chi_{c 10}$ & $\chi_{c 11}$ \\
\hline$\sigma(\mathrm{mb})$ & 3.62 & 20.0 & 6.82 & 15.9 \\
\hline
\end{tabular}

We follow the analysis of [7] to evaluate the fraction of $J / \psi$ 's (in $p p$ collisions) that come from the decays of the $\chi$ and $\psi^{\prime}$. So, the suppression factor $S$ of $J / \psi$ 's produced in the nuclear medium is calculated as:

$S=0.6 \cdot\left(0.92 \cdot S^{J / \psi}+0.08 \cdot S^{\psi^{\prime}}\right)+0.4 \cdot S^{\chi}$. Here $S^{X}$ are the respective suppression factors of the different pure charmonium states $X$ in nuclear matter. The $S^{X}$ are for minimum bias $p A$ collisions within the semiclassical approximation (cf. [ 8]).

The charmonium states are produced as small configurations, then they evolve to their full size. Therefore, if the formation length of the charmonium states, $l_{f}$, becomes larger than the average internucleon distance, one has to take into account the evolution of the cross sections with the distance from the production point [ 5 ].

The formation length of the $J / \psi$ is given by $l_{f} \approx 2 p /\left(m_{\psi^{\prime}}^{2}-m_{J / \psi}^{2}\right)$, where $p$ is the momentum of the $J / \psi$ in the rest frame of the target. For a $J / \psi$ produced at midrapidity at SPS energies, this yields $l_{f} \approx 3 \mathrm{fm}$. Due to the lack of better knowledge, we use the same $l_{f} \approx 3 \mathrm{fm}$ for the $\chi$. For the $\psi^{\prime}$ we use $l_{f} \approx 6 \mathrm{fm}$, because it is not a small object, but has the size of a normal hadron, i.e. the pion. For $E_{l a b}=800 \mathrm{AGeV}$ we get a factor of two for the formation lengths due to the larger Lorentz factor.

However, this has a large impact on the $\psi^{\prime}$ to $J / \psi$-ratio depicted in Fig. 1, which shows the ratio $0.019 \cdot S_{\psi^{\prime}} / S_{J / \psi}$ calculated with (squares $(200 \mathrm{GeV})$ and triangles $\left.(800 \mathrm{GeV})\right)$ and without (crosses) expansion. The factor 0.019 is the measured value in $p p$ collisions, because the experiments do not measure the calculated value $S_{\psi^{\prime}} / S_{J / \psi}$ but $\left(B_{\mu \mu} \sigma\left(\psi^{\prime}\right)\right) /\left(B_{\mu \mu} \sigma(J / \psi)\right)$. $B_{\mu \mu}$ are the branching ratios for $J / \psi, \psi^{\prime} \rightarrow \mu \mu$.

The calculations which take into account the expansion of small wave packages show better agreement with the data (circles) (taken from [ [9]) than the calculation without expansion time, i.e. with immediate $J / \psi$ formation, $l_{f}=0$. We calculated this effect both at $E_{l a b}=200 \mathrm{AGeV}$ and $800 \mathrm{AGeV}$. The data have been measured at different energies ( $E_{l a b}$ $=200,300,400,450,800 \mathrm{GeV}$ and $\sqrt{s}=63 \mathrm{GeV}$ ). One can see that this ratio is nearly constant in the kinematical region of the data, but it decreases at smaller momentum (e.g. $E_{l a b}=200 \mathrm{AGeV}$ and $y<0$ ) due to the larger cross section of the $\psi^{\prime}$.

However, the P-states yield two vastly different cross sections (see Tab. 11) for $\chi_{10}$ and $\chi_{11}$, respectively. This leads to a higher absorption rate of the $\chi_{11}$ as compared to the $\chi_{10}$. This new form of color filtering is predicted also for the corresponding states of other 

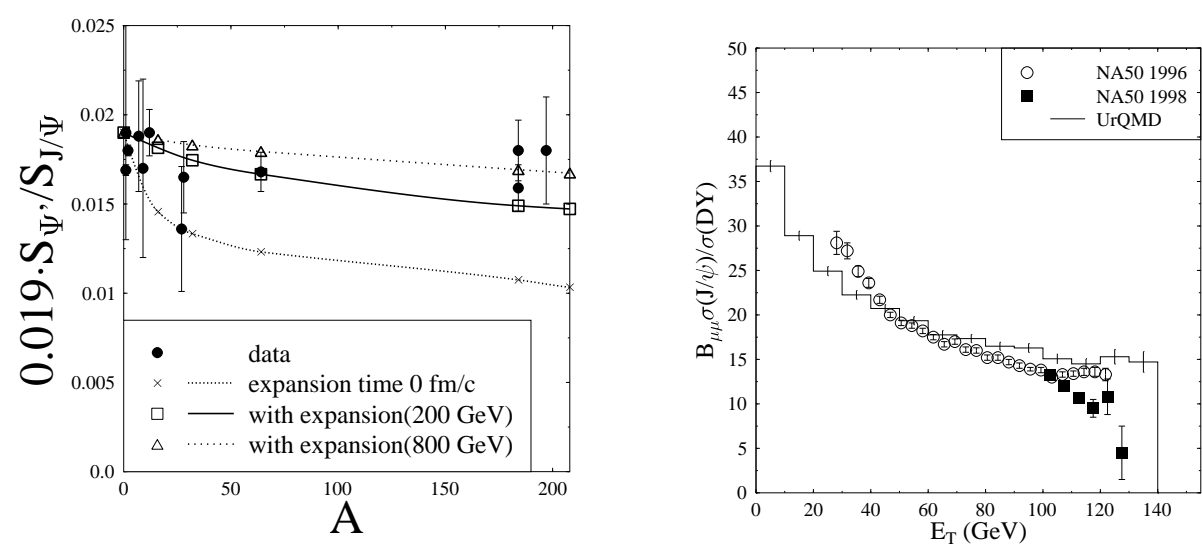

Fig. 1. Left: The ratio $0.019 \cdot S_{\psi^{\prime}} / S_{J / \psi}$ is shown in $p A$ (crosses) in comparison to the data (circles). The squares and the triangles shows the ratio calculated with the expansion of small wave packages. Right: The ratio of $J / \psi$ to Drell-Yan production as a function of $E_{T}$ for $\mathrm{Pb}+\mathrm{Pb}$ at $160 \mathrm{GeV}$.

hadrons; e.g. for the bottomium states which are proposed as contrast signals to the $J / \psi$ 's at RHIC and LHC!

Furthermore it is important to also take into account comoving mesons. Therefore we use the UrQMD model [ 10, 11]. Particles produced by string fragmentation are not allowed to interact with other hadrons - in particular with a charmonium state - within their formation time (on average, $\tau_{F} \approx 1 \mathrm{fm} / \mathrm{c}$ ). However, leading hadrons are allowed to interact with a reduced cross section even within their formation time. The reduction factor is $1 / 2$ for mesons which contain a leading constituent quark from an incident nucleon and $2 / 3$ for baryons which contain a leading diquark.

Figure 11 shows the $J / \psi$ to Drell-Yan ratio as a function of $E_{T}$ for $\mathrm{Pb}+\mathrm{Pb}$ interactions at $160 \mathrm{GeV}$ compared to the NA50 data [12, 13]. The normalization of $B_{\mu \mu} \sigma(J / \psi) / \sigma(\mathrm{DY})=$ 46 in $p p$ interactions at $200 \mathrm{GeV}$ has been fit to $\mathrm{S}+\mathrm{U}$ data within a geometrical model [\#].

The application of this value to our analysis is not arbitrary: the model of Ref. [7] renders the identical $E_{T}$-integrated $J / \psi$ survival probability, $S=0.49$, as the UrQMD calculation for this system. An additional factor of 1.25 [ 14] has been applied to the $\mathrm{Pb}+\mathrm{Pb}$ calculation in order to account for the lower energy, $160 \mathrm{GeV}$, since the $J / \psi$ and Drell-Yan cross sections have different energy and isospin dependencies.

The gross features of the $E_{T}$ dependence of the $J / \psi$ to Drell-Yan ratio are reasonably well described by the model calculation. No discontinuities in the shape of the ratio as a function of $E_{T}$ are predicted by the simulation. The new high $E_{T}$ data [ 13] decreases stronger than the calculation. This could be caused by underestimated fluctuations of the multiplicity of secondaries in the UrQMD model. This occurs, since high $E_{T}$-values are a trigger for very central events with a secondary multiplicity larger than in average [ 15].

\section{Dilepton production}

Beside results from hadronic probes, electromagnetic radiation - and in particular dileptons - offer an unique probe from the hot and dense reaction zone: here, hadronic matter is 
almost transparent. The observed enhancement of the dilepton yield at intermediate invariant masses $\left(M_{e^{+} e^{-}}>0.3 \mathrm{GeV}\right)$ received great interest: it was prematurely thought that the lowering of vector meson masses is required by chiral symmetry restoration (see e.g.[ 16] for a review).

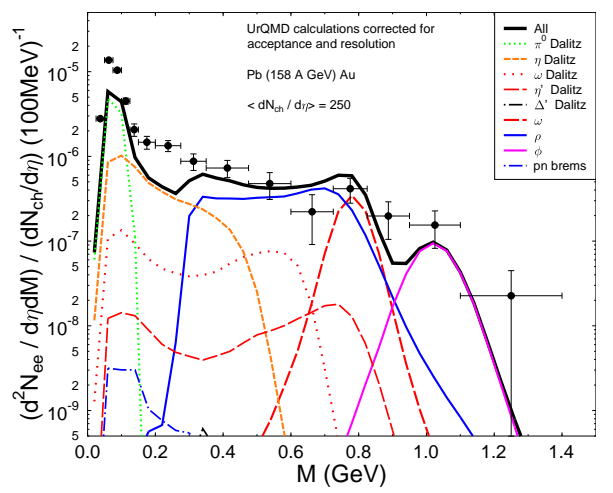

Fig. 2.

Microscopic calculation of the dilepton production in the kinematic acceptance region of the CERES detector for $P b+A u$ collisions at $158 \mathrm{GeV}$. No in-medium effects are taken into account. Plotted data points are taken at CERES in '95.

Fig. 2 2 shows a microscopic UrQMD calculation of the dilepton production in the kinematic acceptance region of the CERES detector for $P b+A u$ collisions at $158 \mathrm{GeV}$. This is compared with the ' 95 CERES data[ 18 . Aside from the difference at $M \approx 0.4 \mathrm{GeV}$ there is a strong enhancement at higher invariant masses. It is expected that this discrepancy at $m>1 \mathrm{GeV}$ could be filled up by direct dilepton production in meson-meson collisions [19] as well as by the mechanism of secondary Drell-Yan pair production proposed in[20].

\section{Strangeness production}

Strange particle yields are most interesting and useful probes to examine excited nuclear matter [21, 22, 23, 24, 25, 26, 27] and to detect the transition of (confined) hadronic matter to quark-gluon-matter. The relative enhancement of strange and especially multistrange particles in central heavy ion collisions with respect to peripheral or proton induced interactions have been suggested as a signature for the transient existence of a QGP-phase [21]. Here the main idea is that the strange (and antistrange) quarks are thought to be produced more easily and hence also more abundantly in such a deconfined state as compared to the production via highly threshold suppressed inelastic hadronic collisions. The relative enhanement of (anti)hyperons has clearly been measured by the WA97 an the NA49 collaboration in $\mathrm{Pb}-\mathrm{Pb}$ collisions as compared to $\mathrm{p}-\mathrm{Pb}$ collisions [ 25, 26]. This data has been investigated within microscopic transport models (e.g. UrQMD [ 10]). In [22, 28] it was shown that within such an appraoch strangeness enhancement is predicted for $\mathrm{Pb}$ $\mathrm{Pb}$ due to rescattering. However, for central $\mathrm{Pb}-\mathrm{Pb}$ collisions the experimentally observed hyperon yields are underestimated by the calculation in [22, 28]. This result seems to confirm the conclusion that a deconfined QGP is formed in $\mathrm{Pb}-\mathrm{Pb}$ collisions at SPS. But in [27, 29] it was shown, that the antihyperon production by multi-mesonic reactions like $n_{1} \pi+n_{2} K \rightarrow \bar{Y}+p$ could drive these rare particles towards local chemical quilibrium with pions, nucleons and kaons on a timescale of $1-3 \mathrm{fm} / \mathrm{c}$. Accordingly this mechanism, which 
is a consequence of detailed balance could provide a convenient explanation for the antihyperon yields at CERN-SPS energies without any need of a deconfined quark-gluon-plasma phase. At the moment such back-reactions cannot be handled within the present transport codes. Therefore the aim for the future will be to find a way to include these processes in microscopic transport models.

\section{Particle ratios}

Ideal gas model calculations have been used for a long time to calculate particle production in relativistic heavy ion collisions, see e.g. [ 30, 31, 32, 33, 34, 35]. Fitting the particle ratios as obtained from those ideal gas calculations to the experimental measured ratios at SIS, AGS and SPS for different energies and different colliding systems yields a curve of chemical freeze-out in the $T-\mu$ plane. Now the question arises, how much the deduced temperature and chemical potentials depend on the model employed. Especially the influence of changing hadron masses and effective potentials should be investigated, as has been done for example in [ 36, 37, 38, 39]. This is of special importance for the quest of a signal of the formation of a deconfined phase, i.e. the quark-gluon plasma. As deduced from lattice data [40], the critical temperature for the onset of a deconfined phase coincides with that of a chirally restored phase. Chiral effective models of QCD therefore can be utilized to give important insights on signals from a quark-gluon plasma formed in heavy-ion collisions.

Therefore we compare experimental measurements for $\mathrm{Pb}+\mathrm{Pb}$ collisions at SPS with the ideal gas calculations and results obtained from a chiral SU(3) model [ 39, 41]. This effective hadronic model predicts a chiral phase transition at $T \approx 150 \mathrm{MeV}$. Furthermore the model predicts changing hadronic masses and effective chemical potentials, due to strong scalar and vector fields in hot and dense hadronic matter, which are constrained by chiral symmetry from the QCD Lagrangean.

In [ 32] the ideal gas model was fitted to particle ratios measured in $\mathrm{Pb}+\mathrm{Pb}$ collisions at SPS. The lowest $\chi^{2}$ is obtained for $T=168 \mathrm{MeV}$ and $\mu_{q}=88.67 \mathrm{MeV}$. Using these values as input for the chiral model leads to dramatic changes due to the changing hadronic masses in hot and dense matter [42] and therefore the freeze-out temperature and chemical potential have to be readjusted to account for the in-medium effects of the hadrons in the chiral model. We call the best fit the parameter set that gives a minimum in the value of $\chi^{2}$, with $\chi^{2}=\sum_{i} \frac{\left(r_{i}^{\text {exp }}-r_{i}^{\text {model }}\right)^{2}}{\sigma_{i}^{2}}$. Here $r_{i}^{\text {exp }}$ is the experimental ratio, $r_{i}^{\text {model }}$ is the ratio calculated in the model and $\sigma_{i}$ represents the error in the experimental data points as quoted in [32]. The resulting values of $\chi^{2}$ for different $T-\mu$ pairs are shown in figure 3. In all calculations $\mu_{s}$ was chosen such that the overall net strangeness $f_{s}$ is zero. The best values for the parameters are $T=144 \mathrm{MeV}$ and $\mu_{q} \approx 95 \mathrm{MeV}$. While the value of the chemical potential does not change much compared to the ideal gas calculation, the value of the temperature is lowered by more than $20 \mathrm{MeV}$. Furthermore Figure 3 shows, that the dropping effective masses and the reduction of the effective chemical potential make the reproduction of experimentally measured particle ratios as seen at CERN's SPS within this model impossible for $T>T_{c}$. Using the best fit parameters a reasonable description of the 

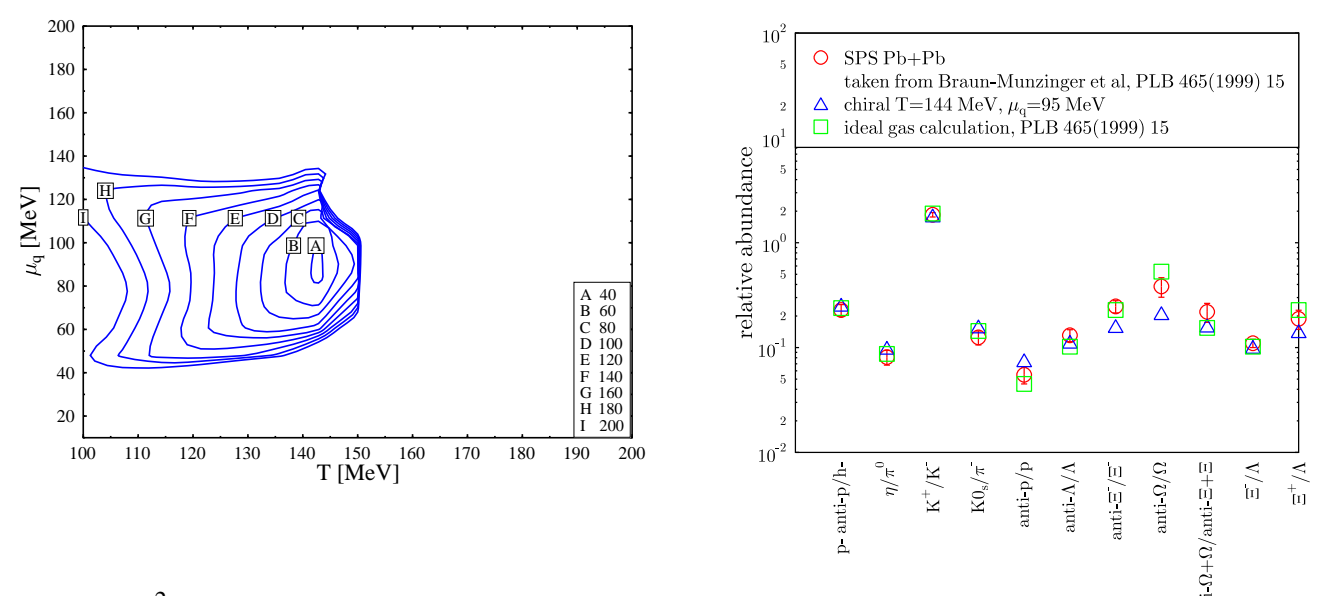

Fig. 3. $\chi^{2}$ (left) and resulting particle ratios compared to ideal gas calculation and data (right for chiral model, data taken from [32]. The best fit parameters are $T=144 \mathrm{MeV}$ and $\mu_{q} \approx 95 \mathrm{MeV}$.

particle ratios used in the fit procedure can be obtained (see fig.3], data from [32]).

We want to emphasize, that in spite of the strong assumption of thermal and chemical equilibrium the obtained values for $T$ and $\mu$ differ significantly depending on the underlying model, i.e. whether and how effective masses and effective chemical potentials are accounted for. Note that we assume implicitly, that the particle ratios are determined by the medium effects and freeze out during the late stage expansion - no flavor changing collisions occur anymore, but the hadrons can take the necessary energy to get onto their mass shall by drawing energy from the fields. Rescattering effects will alter our conclusion but are presumably small when the chemical potentials are frozen.

\section{Collective flow and the EOS}

The in-plane flow has been proposed as a measure of the "softening" of the EoS [ 43], therefore we investigate the excitation function of directed in-plane flow. A three-fluid model with dynamical unification of kinetically equilibrated fluid elements is applied [44]. This model assumes that a projectile- and a target fluid interpenetrate upon impact of the two nuclei, creating a third fluid via new source terms in the continuity equations for energyand momentum flux. Those source terms are taken from energy- and rapidity loss measurements in high energy $p p$-collisions. The equation of state (EoS) of this model assumes equilibrium only in each fluid separately and allows for a first order phase transition to a quark gluon plasma in fluid 1, 2 or 3, if the energy density in the fluid under consideration exceeds the critical value for two phase coexistence. Pure QGP can also be formed in every fluid separately, if the energy density in that fluid exceeds the maximum energy density for the mixed phase. Integrating up the collective momentum in $x$-direction at given rapidity, and dividing by the net baryon number in that rapidity bin, we obtain the so-called directed in-plane flow per nucleon.

Its excitation function (Fig. (4) shows a local minimum at $8 \mathrm{AGeV}$ and rises until a 
maximum around $40 \mathrm{AGeV}$ is reached. Fig. 7 shows the excitation function of directed flow calculated in the three-fluid model in comparison to that obtained in a one-fluid calculation. Due to non-equilibrium effects in the early stage of the reaction, which delay the build-up of transverse pressure[ 45 , the flow shifts to higher bombarding energies. While measurements of flow at AGS [46] have found a decrease of directed flow with increasing bombarding energy, a minimum has so far not been observed.

In a recent investigation of the directed flow excitation functions [ 47] it has been shown, that the directed flow excitation functions are sensitive to the underlying EoS and that a different EoS can predict a slowly and smoothly decrease of the averaged directed flow as a function of bombarding energies. This different behaviour is due to the different phase transitions in the underlying equations of state. While in the two phase EoS based on a $\sigma-\omega$ model for the hadronic phase and a bag model for the deconfined phase a firstorder phase transition occurs, the EoS in [47] provides a continues phase transition of the cross-over type.
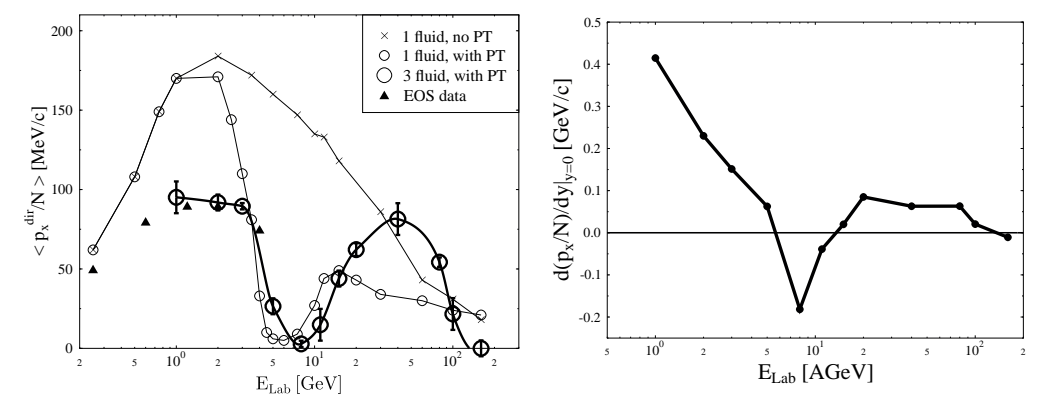

Fig. 4. (Left) Excitation function of transverse flow as obtained from three fluid hydrodynamics with a first order phase transition and (Right) the slope of the directed in-plane momentum per nucleon at midrapidity.

The slope of the directed in-plane momentum per nucleon at midrapidity, $d\left(p_{x} / N\right) / d y$, is shown in Fig. $\bigoplus$ as a function of beam energy. We find a steady decrease of $d\left(p_{x} / N\right) / d y$ up to about top BNL-AGS energy, where the flow around midrapidity even becomes negative due to preferred expansion towards $p_{x} \cdot p_{\text {long }}<0$. At higher energy, $E_{L a b} \simeq 40 \mathrm{~A} \mathrm{GeV}$, the isentropic speed of sound becomes small and we encounter the following expansion pattern : flow towards $p_{x} \cdot p_{\text {long }}<0$ can not build up ! Consequently, $d\left(p_{x} / N\right) / d y$ increases rapidly towards $E_{L a b}=20-40 \mathrm{~A} \mathrm{GeV}$, decreasing again at even higher energy because of the more forward-backward peaked kinematics which is unfavorable for directed flow.

Thus, the $P b+P b$ collisions $(40 \mathrm{GeV}$ ) runs performed recently at the CERN-SPS may provide a crucial test of the picture of a quasi-adiabatic first-order hadronization phase transition at small isentropic velocity of sound.

\section{Collective Flow at RHIC}

Let us now compare the first results on elliptic flow $\left(v_{2}\right)$ at $\sqrt{s_{N N}}=130 \mathrm{GeV}$ as reported by the STAR-Collaboration [ [8] with a string hadronic model simulation: The experimental data indicates a strongly rising $v_{2}$ as a function of $p_{t}$ with an average $v_{2}$ value of $6 \%$ at 
midrapidity and $p_{t}$ approximately $600 \mathrm{MeV}$. While the strong increase of $v_{2}$ with $p_{t}$ has been predicted by the UrQMD model [49] the absolute magnitude of $v_{2}$ at $p_{t}=600 \mathrm{MeV}$ is underpredicted by a factor 3 (cf. fig. 5 ).

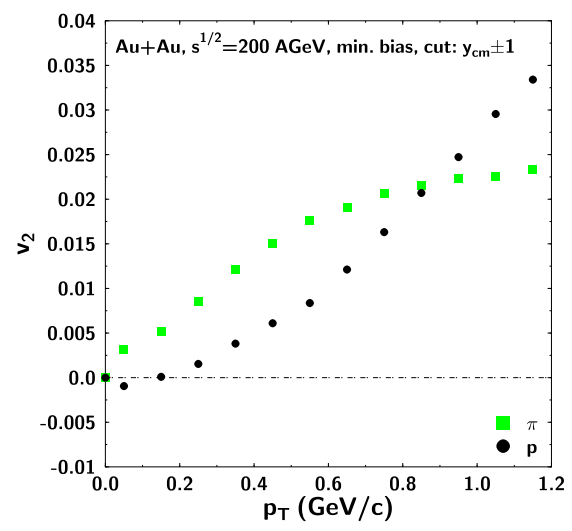

Fig. 5.

Elliptic flow parameter $v_{2}$ at midrapidity as a function of transverse momentum in minimum biased $\mathrm{Au}+\mathrm{Au}$ reactions at $\sqrt{s}=200 \mathrm{AGeV}$

When the formation time of hadrons in the initial strings is strongly reduced (to mimic short mean free paths in the early interaction region) the calculated flow values approach the hydrodynamic limit [ 50, 49] and get in line with the measured elliptic flow values. This shows, that the pressure in the reaction zone is much higher than expected from simple stringlike models and supports the breakdown of pure string hadronic dynamics in the initial stage of Au-Au-collisions at RHIC energies. However, to get a consistent picture and to finally rule out the string hadronic approach the $v_{1}$ values and transverse momentum spectra [ 51] as given by the model calculation need to be exceeded by the experimental data.

\section{Insights from quark molecular dynamics}

Further insights about the possible formation of deconfined matter can be obtained from the Quark Molecular Dynamics Model (qMD) [ 52] which explicitly includes quark degrees of freedom. The qMD can provide us with detailed information about the dynamics of the quark system and the parton-hadron conversion. Correlations between the quarks clustering to build new hadrons can be studied [53].

Figure 6 shows (for $\mathrm{S}+\mathrm{Au}$ collisions at SPS energies of $200 \mathrm{GeV} / \mathrm{N}$ ) the number distribution for the mean path travelled by quarks forming a hadron (a) from the same initial hadron (solid line) and (b) from different initial hadrons (dotted line).

A measure of the relative mixing within the quark system and thus for thermalization is the relative number of hadrons formed by quarks from the same initial hadron correlation versus hadrons formed by quarks from different initial hadron correlations. This ratio is $r=0.574 \pm 0.008$ for the $\mathrm{S}+\mathrm{Au}$ collision. Since a value of $r=1$ would indicate complete rearrangement of quarks and thus complete loss of correlations in the quark system, one would expect a much larger value of $r$, considering the presumed transition to the quarkgluon plasma in $\mathrm{Pb}+\mathrm{Pb}$ collisions at $160 \mathrm{GeV} / \mathrm{N}$, 


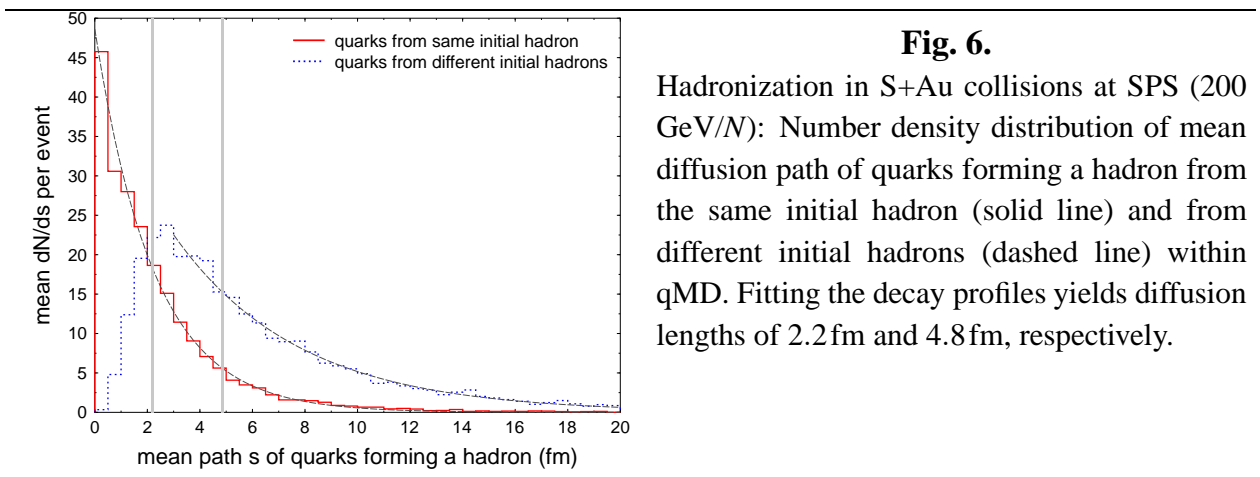

\section{Outlook}

The latest data of CERN/SPS on flow, electro-magnetic probes, strange particle yields (most importantly multistrange (anti-)hyperons) and heavy quarkonia will be interesting to follow closely. Simple energy densities estimated from rapidity distributions and temperatures extracted from particle spectra indicate that initial conditions could be near or just above the domain of deconfinement and chiral symmetry restoration. Still the quest for an unambiguous signature remains open.

Directed flow has been discovered - now a flow excitation function, filling the gap between $10 \mathrm{AGeV}$ (AGS) and $160 \mathrm{AGeV}$ (SPS), will be extremely interesting: look for the softening of the QCD equation of state in the coexistence region. The investigation of the physics of high baryon density (e.g. partial restoration of chiral symmetry via properties of vector mesons) has been pushed forward by the $40 \mathrm{GeV}$ run at SPS. Also the excitation function of particle yield ratios $(\pi / p, d / p, K / \pi \ldots)$ and, in particular, multistrange (anti-)hyperon yields, can be a sensitive probe of physics changes in the EoS. The search for novel, unexpected forms of matter, e.g. hypermatter, strangelets or even charmlets is intriguing. Such exotic QCD multi-meson and multi-baryon configurations would extend the present periodic table of elements into hitherto unexplored dimensions. A strong experimental effort should continue in that direction.

Now we have entered the exciting RHIC era, where the predicted deconfined and chirally restored phase should be formed and live long enough to produce clear and unambigious signals of it's existence. The LHC-program will top this scientific endeavour in 4 years.

\section{Acknowledgments}

This work was supported by DFG, GSI, BMBF, Graduiertenkolleg Theoretische und Experimentelle Schwerionenphysik, the A. v. Humboldt Foundation, and the J. Buchmann Foundation.

\section{References}


1. T. Matsui and H. Satz, Phys. Lett. B178, 416 (1986).

2. D. Kharzeev, Nucl. Phys. A610, 418c (1996).

3. L. Gerland, L. Frankfurt, M. Strikman, H. Stöcker, and W. Greiner, Phys. Rev. Lett. 81, 762 (1998).

4. R. L. Anderson et al., Phys. Rev. Lett. 38, 263 (1977).

5. G. R. Farrar, L. L. Frankfurt, M. I. Strikman, and H. Liu, Phys. Rev. Lett. 64, 2996 (1990).

6. L. Frankfurt, W. Koepf, and M. Strikman, Phys. Rev. D54, 3194 (1996).

7. D. Kharzeev, C. Lourenco, M. Nardi, and H. Satz, Z. Phys. C74, 307 (1997).

8. C. Gerschel and J. Hufner, Phys. Lett. B207, 253 (1988).

9. C. Lourenco, Nucl. Phys. A610, 552c (1996).

10. S. A. Bass et al., Prog. Part. Nucl. Phys. 41, 225 (1998).

11. C. Spieles, R. Vogt, L. Gerland, S. A. Bass, M. Bleicher, H. Stocker and W. Greiner, Phys. Rev. C60 (1999) 054901 [hep-ph/9902337].

12. A.Romana et al., in Proceedings of the XXXIIIrd Rencontres de Moriond, March 1998, Les Arcs, France.

13. M. C. Abreu et al., Phys. Lett. B477, 28 (2000).

14. R. Vogt, Phys. Rept. 310, 197 (1999).

15. A. Capella, E. G. Ferreiro, and A. B. Kaidalov, hep-ph/0002300 (2000).

16. V. Koch, Int. Jour. Mod. Phys. E6 (1997) 203.

17. W. Cassing, E. L. Bratkovskaya, R. Rapp, and J. Wambach, Phys. Rev. C57 (1998) 916

18. G. Agakishiev et al., Phys. Lett. B402 (1998) 405.

19. G. Q. Li and C. Gale, Phys. Rev. C58 (1998) 2914.

20. C. Spieles et al., Eur. Phys. J. C5 (1998) 349

21. J. Rafelski, B. Müller Phys. Rev. Lett.48, (1982) 1066; (E) 56 (1986) 2334; P. Koch, B. Müller, J. Rafelski Phys. Rep. 142, (1986) 167; P. Koch, B. Müller, H. Stöcker, W. Greiner Mod. Phys. Lett. A3, (1988) 737

22. S. Soff, S. A. Bass, M. Bleicher, L. Bravina, M. Gorenstein, E. Zabrodin, H. Stöcker, W. Greiner Phys. Lett. B471, (1999) 89 and refs. therein

23. P. Senger, H. Ströbele J. Phys. G25, (1999) R59

24. R. Stock Phys. Lett.B456, (1999) 277

25. E. Andersen et al.(WA97 collaboration) Phys. Lett. B433, (1998) 209; S. Margetis et al.(NA49 collaboration) J. Phys. G 25, (1999) 189

26. F. Sikler et al.(NA49 collaboration) Nucl. Phys. A661, (1999)

27. C. Greiner, S. Leupold nucl-th/0009036

28. S. Soff et al., J. Phys. G in print, nucl-th/0010103.

29. C. Greiner, nucl-th/0011026.

30. D. Hahn and H. Stöcker, Nucl. Phys. A452, 723 (1986).

31. D. Hahn and H. Stöcker, Nucl. Phys. A476, 718 (1988).

32. P. Braun-Munzinger, J. Heppe, and J. Stachel, Phys. Lett. B 465, 15 (1999).

33. J. Rafelski and J. Letessier, nucl-th/9903018 (1999).

34. F. Becattini, J. Cleymans, A. Keranen, E. Suhonen, and K. Redlich, hep-ph/0002267 (2000). 
35. G. D. Yen and M. I. Gorenstein, Phys. Rev. C59, 2788 (1999).

36. H. Stöcker and W. Greiner, Z. Phys. A 286, 121 (1978).

37. J. Theis, G. Graebner, G. Buchwald, J. A. Maruhn, W. Greiner, H. Stöcker and J. Polonyi, Phys. Rev. D28 (1983) 2286.

38. J. Schaffner, I. N. Mishustin, L. M. Satarov, H. Stöcker, and W. Greiner, Z. Phys. A341, 47 (1991).

39. D. Zschiesche, P. Papazoglou, S. Schramm, C. Beckmann, J. Schaffner-Bielich, H. Stöcker, and W. Greiner, Springer Tracts in Modern Physics 163, 129 (2000).

40. F. Karsch, hep-lat/9903031 (1998).

41. P. Papazoglou, D. Zschiesche, S. Schramm, J. Schaffner-Bielich, H. Stöcker, and W. Greiner, Phys. Rev. C 59, 411 (1999).

42. D. Zschiesche, L. Gerland, S. Schramm, J. Schaffner-Bielich, H. Stöcker and W. Greiner, nucl-th/0007033.

43. D. H. Rischke, Y. Pürsün, J.A. Maruhn, H. Stöcker, W. Greiner, Heavy Ion Physics 1 (1995) 309.

44. J. Brachmann, A. Dumitru, J.A. Maruhn, H. Stöcker, W. Greiner, D.H. Rischke, Nucl. Phys. A619 (1997) 391.

45. H. Sorge, Phys. Rev. Lett. 78, 2309 (1997)

46. H. Liu et al. (E895 Collaboration), Nucl. Phys. A638, 451c (1998)

47. Y. B. Ivanov, E. G. Nikonov, W. Noerenberg, A. A. Shanenko and V. D. Toneev, nucl-th/0011004.

48. K. H. Ackermann et al. [STAR Collaboration], nucl-ex/0009011.

49. M. Bleicher and H. Stöcker, hep-ph/0006147.

50. P. Huovinen, priv. comm.;

P. F. Kolb, J. Sollfrank and U. Heinz, Phys. Lett. B459 (1999) 667 [nucl-th/9906003].

51. M. Bleicher et al., Phys. Rev. C62 (2000) 024904 [hep-ph/9911420].

52. M. Hofmann, S. Scherer, M. Bleicher, L. Neise, H. Stöcker, and W. Greiner, Phys. Lett. B478 (200) 161

53. S. Scherer, M. Hofmann, M. Bleicher, L. Neise, H. Stöcker, and W. Greiner, N. Journ.Pंhys. to be publ. 